\title{
WEB WATCH
}

\section{Research resource \\ - http://www.cancer- portfolio.org}

A powerful new tool provides instant access to information about current cancer research projects that are supported by cancer funding organizations in the United States and the United Kingdom.

The International Cancer Research Portfolio (ICRP) is a public database that holds nearly 13,000 records of current cancer research awards. This site is a centra searchable database, which allows visitors to find

information about actively

funded research. The database has a simple and useful keyword-based search engine. Search results provide clear, concise information on the titles, principle investigators, institutions, funding organizations and award codes of all funded research projects. Title links take the visitor to detailed abstracts of each project.

The ICRP was created by the Common Scientific Outline (CSO) Partners eight United States cancer funding organizations and 15 members of the Nationa Cancer Research Institute of the United Kingdom - to share information about the research that they support. The CSO was established by the National Cancer Institute in September 2000 to encourage international collaboration and to compare the types of research funded by each country.

The web site is designed for use by cancer researchers, and funding organizations, health-care policy makers, health-care professionals, and patients and their advocates. Researchers can use the site to identify scientists that do similar work, or to find contacts for multidisciplinary collaborations. The CSO also hopes that funding organizations and government/policy officials will use the site to increase their current research knowledge and to plan for future research efforts.

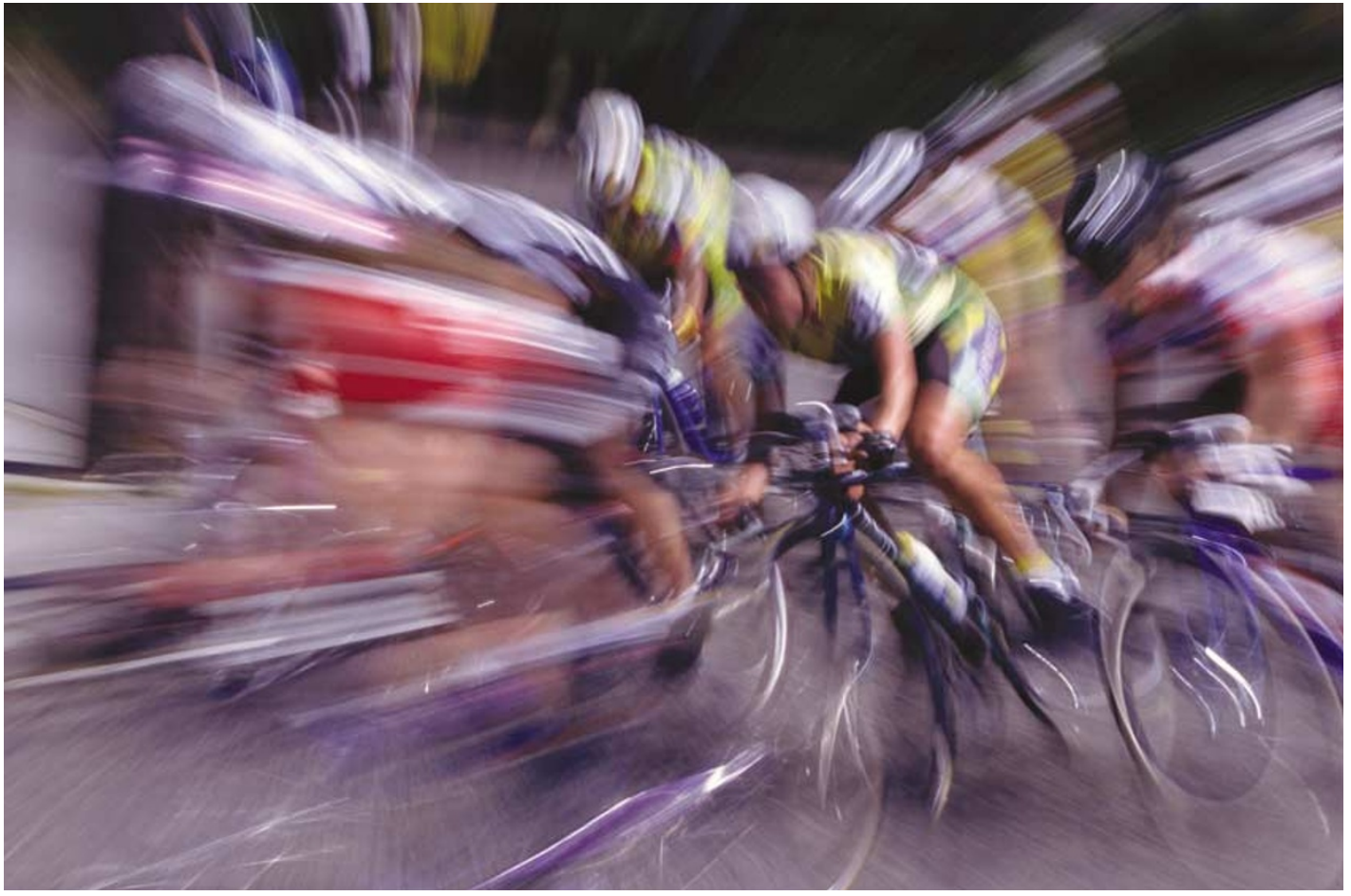

METASTASIS

\section{Keep moving}

The processes involved in tumour-cell metastasis are complex and, in many cases, unresolved, but the most basic feature is cell movement - away from the primary tumour through the extracellular matrix (ECM). Cell motility involves remodelling of the cytoskeleton. As RHO-family GTPases are known to regulate cytoskeletal proteins such as actin - through the RHO kinase (ROCK) family - and are often overexpressed in tumours, Sahai and Marshall looked at the role of this signalling pathway in the motility of tumour cell lines.

The movement of cell lines in a three-dimensional ECM-like matrix was assessed in response to TAT-C3, which inactivates RHO proteins, and Y27632, which inhibits ROCK proteins. The invasive ability of some cell lines (such as A375m2 metastatic melanoma) was almost completely blocked by either inhibitor, whereas other cell lines (such as BE colon carcinoma) were not affected. The authors noted a correlation between the effects of these inhibitors and the morphology of the invading cells. Those cell lines that were susceptible to inhibition of RHO or ROCK had rounded cells with membrane blebs. By contrast, cell lines such as BE had cells that were elongated with membrane spikes. These morphologies were confirmed in vivo by studying tumour biopsies of green fluorescent protein (GFP)expressing tumour cells injected subcutaneously in nude mice.

To show that this correlation is causal, the effects of modulating RHO-ROCK signalling on tumour-cell morphology were examined. $\mathrm{A} 375 \mathrm{~m} 2$ cells treated with TAT-C 3 or Y27632 lost their membrane blebs and developed membrane protrusions. In parental nonmetastatic A375 cells, which are less rounded, overexpression or constitutive activation of RHO or ROCK proteins increased cell rounding. BE cells transfected with constitutively active RHOA or
ROCK1 lost their elongated morphology and became more rounded. Therefore, RHO-ROCK signalling is necessary and sufficient for a round morphology.

Finally, Sahai and Marshall looked at the mechanism of cell motility. In contrast to cells that move in an elongated manner, rounded $\mathrm{A} 375 \mathrm{~m} 2$ cells did not show localization of phosphatidylinositol3,4,5-trisphosphate to the leading edge or polarization of the Golgi apparatus. Instead, rounded cells had localized membrane patches of ezrin orientated in the direction of cell movement, and interfering with ezrin function inhibited invasive ability. Ezrin links cell-matrix interactions to the cytoskeleton, so movement occurs in response to polarized cell adhesions that 'drag' the cell in one direction.

Interestingly, the movement of rounded $\mathrm{A} 375 \mathrm{~m} 2$ cells was shown not to depend on proteolytic degradation of the matrix. Furthermore, when extracellular proteolysis was blocked, cells that would normally move in an elongated manner (such as BE cells), with no requirement for RHO-ROCK signalling, began to move in a rounded RHO-ROCK-dependent way. So, the authors showed that for some tumour cells, invasion is only blocked when both proteolysis and ROCK signalling are inhibited. Future therapeutic strategies to block metastasis will need to take into account the relative roles of these two modes of movement for different tumour types and their interconvertibility. This will require the discovery of histological markers that identify tumour cells with rounded or elongated invasive morphologies.

Kirsty Minton

\section{(9) References and links}

ORIGINAL RESEARCh PAPER Sahai, E. \& Marshall, C. J. Differing modes of tumour-cell invasion have distinct requirements for Rho/ROCK signalling and extracellular proteolysis Nature Cell Biol 6 Jul 2003 (doi:10 1038/ncb1019) FURTHER READING Sahai, E.\& Marshall, C. J. RHO-GTPases and cancer. Nature Rev. Cancer 2, 133-142 (2002) 